\title{
The time course of dysphagia following traumatic cervical spinal cord injury: a prospective cohort study
}

\author{
Tetsuo Hayashi ${ }^{1,2} \cdot$ Yuichi Fujiwara $^{3} \cdot$ Hiroaki Sakai $^{2} \cdot$ Kensuke Kubota $^{1,2} \cdot$ Osamu Kawano $^{2} \cdot$ Eiji Mori $^{2}$. \\ Tsuneaki Takao ${ }^{2} \cdot$ Muneaki Masuda $\left(\mathbb{D}^{2} \cdot\right.$ Yuichiro Morishita ${ }^{2} \cdot$ Takeshi Maeda $^{2}$
}

Received: 27 March 2019 / Revised: 27 July 2019 / Accepted: 30 July 2019 / Published online: 28 August 2019

(c) The Author(s), under exclusive licence to International Spinal Cord Society 2019

\begin{abstract}
Study design Prospective cohort study.

Objectives To elucidate serial changes in dysphagia and elucidate the critical period for dysphagia following acute traumatic cervical spinal cord injury (CSCI).

Setting Spinal Injuries Center, Fukuoka, Japan.

Methods We prospectively examined individuals with acute traumatic CSCI admitted within 2 weeks after injury. Severity of dysphagia was evaluated using both the dysphagia severity scale (DSS) and functional oral intake scale (FOIS) at 2 weeks, 1 month, 2 months, and 3 months after injury. Condition of oral intake before injury was assessed by history taking. American Spinal Injury Association (ASIA) impairment scale grade and motor scores were also assessed at the same timepoints, and the correlation between dysphagia and paresis was analyzed.

Results Sixty-five individuals with CSCI were assessed consecutively for 3 months after injury. Swallowing function, evaluated using both the DSS and FOIS, was significantly decreased at 2 weeks after injury, but significantly improved thereafter. Significant correlations between severity of dysphagia (DSS and FOIS scores) and motor scores were found at 2 weeks after injury $\left(r_{\mathrm{s}}=0.66\right.$ and $0.61 ; p<0.001$ and $p<0.001$, respectively), indicating that individuals with lower motor scores had more severe swallowing dysfunction.

Conclusions Dysphagia occurred immediately after injury, but gradually improved over time. Individuals with more severe paralysis had significantly more severe dysphagia. Special attention for dysphagia should be paid to individuals with severe paralysis in acute phase.
\end{abstract}

\section{Introduction}

Pneumonia following cervical spinal cord injury (CSCI) can be a life-threatening condition [1]. In particular, aspiration pneumonia remains a major cause of further morbidity and mortality [2,3] and is an economic burden to treat [4].

Tetsuo Hayashi

tetsuo884hayashi@yahoo.co.jp

1 Department of Rehabilitation Medicine, Japan Organization of Occupational Health and Safety, Spinal Injuries Center, Fukuoka, Japan

2 Department of Orthopaedic Surgery, Japan Organization of Occupational Health and Safety, Spinal Injuries Center, Fukuoka, Japan

3 Nursing Department, Japan Organization of Occupational Health and Safety, Spinal Injuries Center, Fukuoka, Japan
Swallowing dysfunction, which may cause aspiration pneumonia, is one of the most important complications when we treat individuals with CSCI. Early detection of dysphagia is necessary to secure sufficient nutrition and avoid aspiration pneumonia.

The relationship between CSCI and dysphagia has been reported, and the risk factors for dysphagia development have been identified [5-9]. A recent meta-analysis reported that significant risk factors include age, severity of injury, level of injury, tracheostomy, cough, voice quality, need for bronchoscopy, pneumonia, mechanical ventilation, nasogastric tubes, comorbid injury, and cervical surgery [10].

While the risk factors have been reported, to our knowledge, no previous study has reported the serial changes in dysphagia following CSCI. It is very important to understand the clinical course of swallowing dysfunction in order to take special care at meals during the critical period for not only dysphagia but also aspiration 
pneumonia. We hypothesized that dysphagia frequently occurs immediately after CSCI. The purpose of this study was to examine serial changes in dysphagia and elucidate the critical period for dysphagia in individuals with CSCI.

\section{Methods}

\section{Participants}

Between October 2015 and May 2017, 91 individuals with acute traumatic CSCI were admitted to the Spinal Injuries Center in Fukuoka prefecture, Japan (69 men, 22 women; median age, 68 years [interquartile range, 59-76 years]). Classification of spinal injuries according to the American Spinal Injury Association (ASIA) impairment scale was as follows: grade $\mathrm{A}$ in 18 individuals, grade $\mathrm{B}$ in 7 , grade $\mathrm{C}$ in 28 , grade $\mathrm{D}$ in 23 , and no paresis in 15 . A prospective cohort study was conducted in those participants.

This study included individuals with traumatic CSCI who were admitted within 14 days of injury and were followed consecutively for at least 3 months. The following comprised the exclusion criteria: brain injury, cerebral infarction, and switching hospitals due to deterioration of the general condition. In addition, patients discharged to their homes due to recovery of their neurologic condition at $<3$ months were also excluded.

\section{Evaluation}

Swallowing function and neurologic condition were prospectively examined. Swallowing function was classified using the dysphagia severity scale (DSS) [11] and functional oral intake scale (FOIS) [12]. The DSS evaluates the condition of aspiration or dysphagia using the following scores: 1, saliva aspiration; 2, food aspiration; 3, water aspiration; 4, occasional aspiration; 5, oral problems; 6, minimum problems; and 7, within normal limits. The FOIS evaluates the functional intake of food or liquid using the following scores: 1 , nothing by mouth; 2 , tube dependent with minimal attempts of food or liquid; 3 , tube dependent with consistent oral intake of food or liquid; 4 , total oral diet of a single consistency; 5, total oral diet with multiple consistencies but requiring special preparation or compensations; 6 , total oral diet with multiple consistencies without special preparation but with specific food limitations; and 7, total oral diet with no restrictions. We also used fiberoptic endoscopic evaluation of swallowing (FEES) or videofluoroscopy (VFS) to make a detailed evaluation and decide the classification of DSS, when patients were categorized as scale 4 or less of DSS. Both DSS and FOIS were discussed and determined by TH (1st author) and YF (2nd author) in reference to the objective results of FEES or VFS. Both
DSS and FOIS scores were evaluated at 2 weeks, 1 month, 2 months, and 3 months after injury. Condition of oral intake before injury was assessed by history taking using both the DSS and FOIS. No treatment by Speech and language therapist was performed, however, a certified nurse in dysphagia nursing (YF) supervised feeding of all patients with dysphagia.

In addition, ASIA impairment scale [13] and motor scores (range, 0-100) were examined to evaluate individuals' neurologic status at the same timepoints. Also, level of injury detected using MRI and the presence of tracheostomy were examined.

\section{Statistical analysis}

Correlations between severity of dysphagia (DSS and FOIS scores) and motor scores were assessed using Spearman correlation coefficients. When examining serial changes in dysphagia, the Wilcoxon signed-rank test was used to analyze differences among timepoints (before injury and 2 weeks, 1 month, 2 months, and 3 months after injury). Correcting with Bonferroni inequality, a $p$ value $<0.01$ $(0.05 / 5)$ was considered statistically significant. Statistical analyses were performed using JMP 13 (SAS Institute Inc., Cary, NC, USA) computer software.

\section{Results}

Of the 91 individuals admitted to our hospital within 2 weeks after injury, 13 were excluded due to switching hospitals within 3 months (deterioration of general condition in six, dementia or mental problem in four, transfer to local hospital in three). In addition, 13 individuals could not be followed for 3 months. Finally, the remaining 65 individuals were followed consecutively for 3 months after injury (Fig. 1).

Table 1 shows the demographic data of the 65 individuals in this study. Median age was 67 years old and interquartile range was 60-73 years old. In terms of level of injury, 28 individuals (43\%) had spinal injury at C3 to 4,10

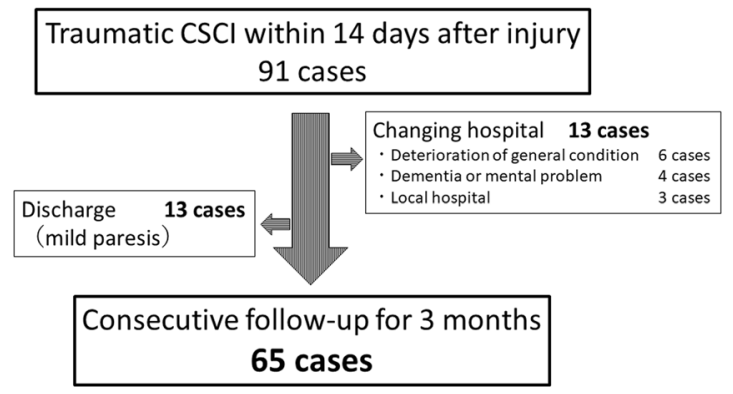

Fig. 1 Flowchart of the study. CSCI cervical spinal cord injury 
Table 1 Summary of demographic data of 65 patients at two weeks after injury

\begin{tabular}{ll}
\hline Age (year) & $67(60-73)$ \\
Sex (female/male) & $14 / 51$ \\
DSS & $5(4-6)$ \\
FOIS & $6(5-6)$ \\
Tracheostomy & $8(12)$ \\
Motor score & $46(19-73)$ \\
ASIA impairment scale (\%) & \\
A & $14(22)$ \\
B & $1(2)$ \\
C & $23(35)$ \\
D & $22(34)$ \\
E & $5(8)$ \\
Level of injury $(\%)$ & \\
C1/2 & $4(6)$ \\
C2/3 & $2(3)$ \\
C3/4 & $28(43)$ \\
C4/5 & $10(15)$ \\
C5/6 $6 / 7$ & $8(12)$ \\
C7/T1 & $10(15)$ \\
\hline
\end{tabular}

$(15 \%)$ at $\mathrm{C} 4$ to $5,10(15 \%)$ at $\mathrm{C} 6$ to $7,8(10 \%)$ at $\mathrm{C} 5$ to 6,4 $(6 \%)$ at $\mathrm{C} 1$ to $2,3(5 \%)$ at $\mathrm{C} 7$ to $\mathrm{T} 1$, and $2(3 \%)$ at $\mathrm{C} 2$ to 3.

\section{Serial changes in dysphagia}

Average DSS score of the 65 individuals was significantly decreased after injury compared with before injury; however, it significantly increased for 2 months after injury, indicating that swallowing function was decreased immediately after injury, but gradually improved thereafter (Fig. 2a). Similarly, average FOIS score was significantly decreased after injury compared with before injury; however, it significantly increased at each time point after injury, demonstrating that functional oral intake was decreased immediately after injury, but also gradually improved thereafter (Fig. 2b). When we looked at serial changes in each ASIA impairment scale, both DSS and FOIS in each scale improve gradually after injury, whereas the average scores of DSS and FOIS in complete paralysis showed lower than those in incomplete paresis in every time point (Fig. 2c, d). When evaluating aspiration (DSS score $\leq 4), 22$ individuals $(35 \%)$ suffering from aspiration at 2 weeks after injury decreased to 11 individuals (17\%) at 3 months. Similarly, when evaluating tube dependency (FOIS score $\leq 3$ ), 11 individuals $(17 \%)$ who were tube dependent for nutrition at 2 weeks after injury decreased to 3 individuals (5\%) at 3 months.

\section{Correlation between severity of dysphagia and motor scores at $\mathbf{2}$ weeks after injury}

A significant correlation was identified between DSS and motor scores $\left(r_{\mathrm{s}}=0.67\right.$ and $p<0.001$; Fig. 3a). A significant correlation was also found between FOIS and motor scores $\left(r_{\mathrm{s}}=0.61\right.$ and $p<0.001$; Fig. 3b). These findings indicate that individuals with more severe paresis had more severe dysphagia and less functional oral intake.

\section{Discussion}

The incidence of dysphagia in several studies has been reported to be between 7 and $51 \%$ [3, 5, 8, 10, 14-16]. One of the reasons for this wide range is that individuals with CSCI have various degrees of dysphagia, making it difficult to set a definition for dysphagia. Another reason may be differences in the time of dysphagia evaluation, as swallowing dysfunction can vary over time after injury [17]. The current study confirmed this varying degree of dysphagia from before injury to 3 months after injury.

It is very important to recognize not only the incidence of dysphagia but also its natural course following CSCI. To the best of our knowledge, no previous study has demonstrated this varying degree of dysphagia over time after injury. We found deterioration in swallowing function immediately after CSCI; however, the clinical course of dysphagia showed gradual improvement in most cases. Some individuals still suffered from aspiration and were tube dependent for nutrition; however, all three individuals who were still tube dependent at 3 months had a tracheostomy, which is one of the known risk factors for dysphagia $[3,5,15]$.

Several studies have reported that the level of injury and severity of paralysis have a significant relationship with dysphagia $[5,14,16]$. We previously reported in another series that ASIA impairment scale grade A or B was a significant risk factor for dysphagia according to logistic regression analysis of 298 cases [5]. The current study found a significant correlation between motor scores and severity of dysphagia, indicating more severe dysphagia in individuals with more severe paresis in the acute phase.

This study is not without limitations. Firstly, we followed the individuals for only 3 months from the acute to subacute phase, which is a relatively short period. Studies with longer follow-up would be needed to investigate dysphagia from the subacute to chronic phase. Second, patients with more severe injuries which may have dysphagia might have been excluded because they changed hospital due to deterioration of their conditions. Third, as patients with mild paresis who discharged from our hospital also could not followed, a bias in the population of this study should be acknowledged. 

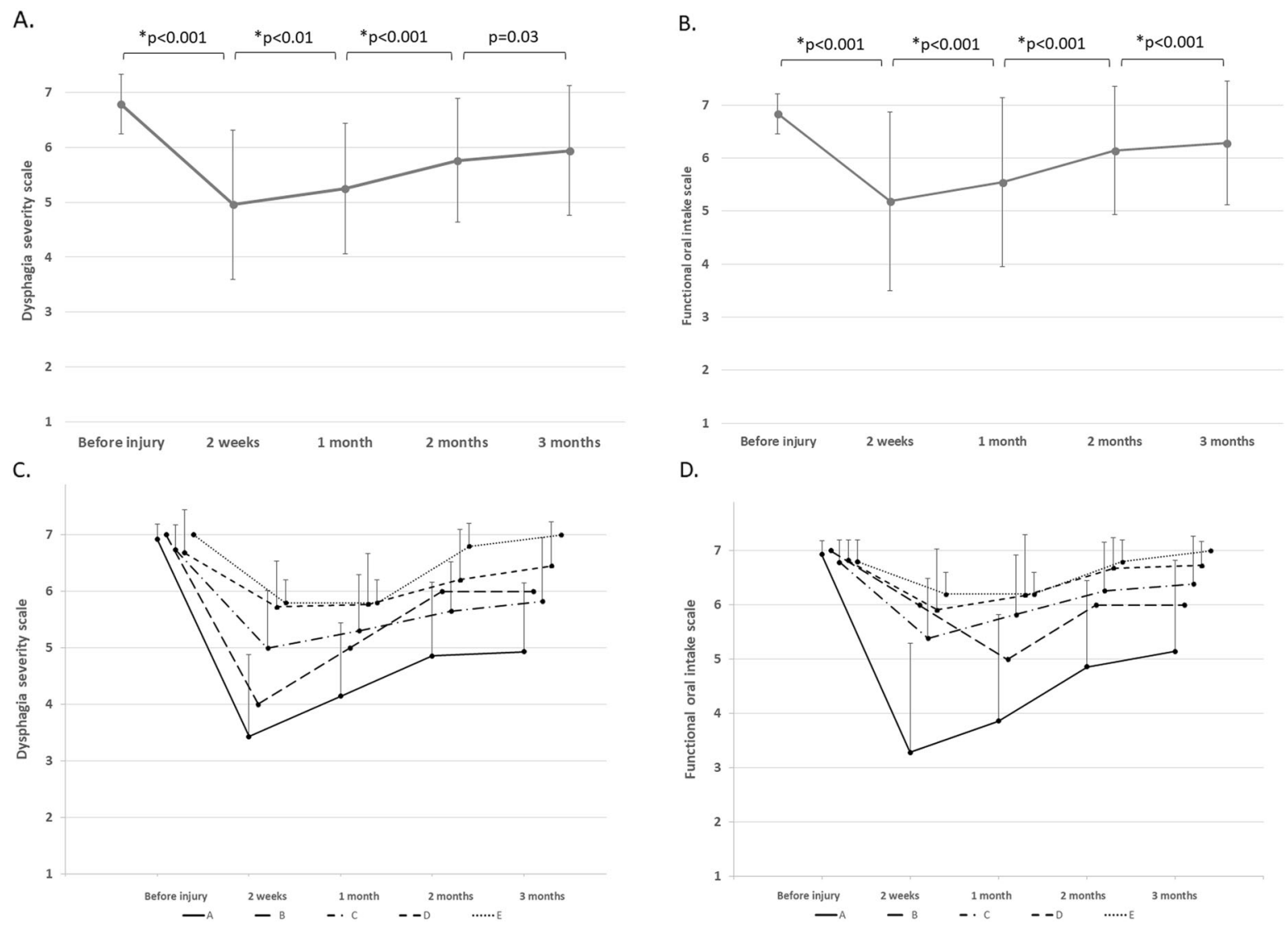

Fig. 2 Serial changes of dysphagia. The dots and bars indicated means and standard deviations. The number of patients with ASIA impairment scale A, B, C, D, and E were 14, 1, 23, 22, and 5, respectively. a Serial changes in dysphagia severity scale score. $\mathbf{b}$ Serial changes in

$$
\text { D. }
$$

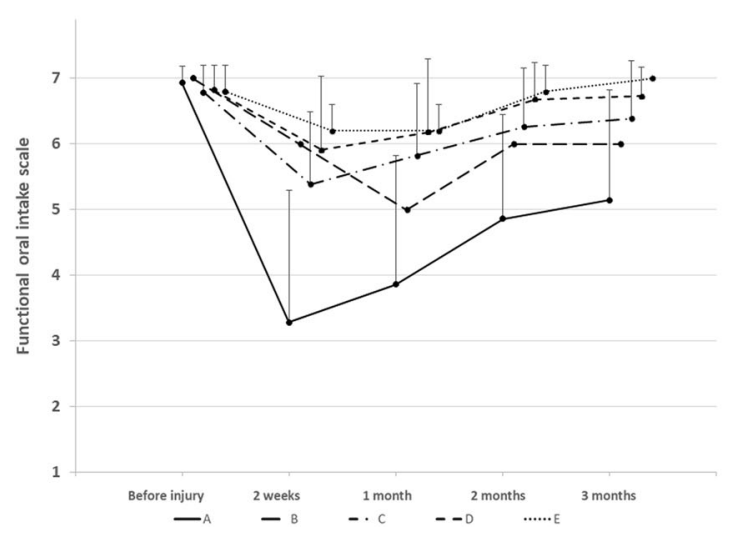

functional oral intake scale score. c Serial changes of dysphagia severity scale in each ASIA impairment scale. d Serial changes of functional oral intake scale in each ASIA impairment scale

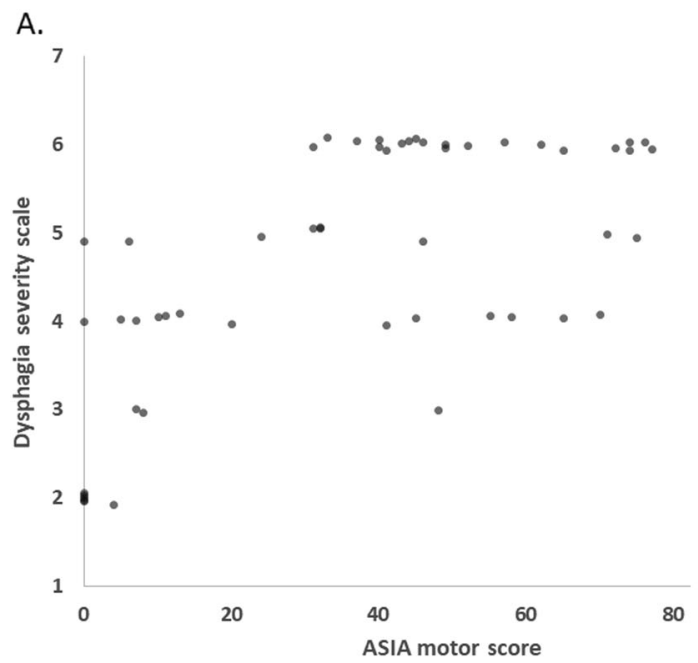

B.

Fig. 3 a Correlation between dysphagia severity scale and motor scores at 2 weeks after injury. Overall 65 individuals were included in this figure although some dots were overlapped. ASIA, American Spinal Injury Association. b Correlation between functional oral intake

When treating individuals with CSCI, we need to pay attention to dysphagia, particularly in the acute phase, especially in two weeks after injury. In addition, individuals

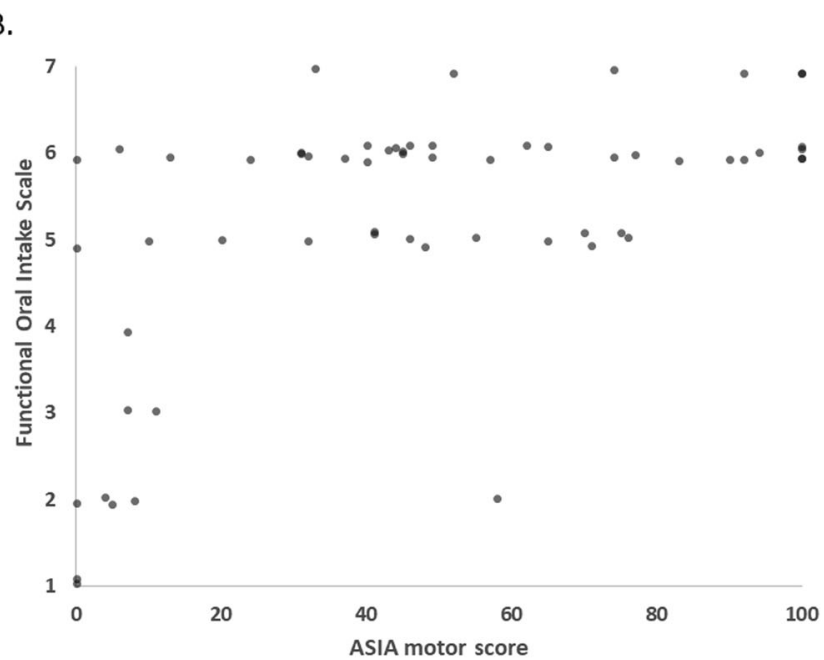

scale and motor scores at 2 weeks after injury. Overall 65 individuals were included in this figure although some dots were overlapped. ASIA, American Spinal Injury Association

with severe paralysis (i.e. low motor scores, and low grade of ASIA impairment scale) should be closely monitored at meals and be considered to treat by direct and/or indirect 
training for eating to avoid aspiration, which may lead to pneumonia and pulmonary dysfunction.

\section{Data availability}

The data sets generated and/or analyzed during the current study are not publicly available due to the inclusion of private information of patients but are available from the corresponding author on reasonable request.

Acknowledgements Works of TH have been supported by a Grant-inAid for Scientific Research (C) from The Japanese Ministry of Education, Culture, Sports, Science and Technology.

Author contributions TH was responsible for designing the study, writing the protocol and report, extracting and analyzing data, screening potential eligible studies, interrupting results, updating reference lists, and creating "Summary of Findings" tables. YF was responsible for conducting the literature search, extracting and analyzing data, and screening potential eligible studies. HS contributed to designing the study. KK contributed to designing the study and interpreting results. OK contributed to interpreting results. EM contributed to interpreting results. TT contributed to interpreting results. $\mathrm{MM}$ contributed to interpreting results. YM contributed to interpreting results. TM contributed to interpreting results and providing feedback on the report.

\section{Compliance with ethical standards}

Conflict of interest The authors declare that they have no conflict of interest.

Ethics statement The study protocol was approved by the Institutional Review Board of the Spinal Injuries Center, and all participants provided written informed consent before participating in the study.

Publisher's note: Springer Nature remains neutral with regard to jurisdictional claims in published maps and institutional affiliations.

\section{References}

1. Grossman RG, Frankowski RF, Burau KD, Toups EG, Crommett JW, Johnson MM, et al. Incidence and severity of acute complications after spinal cord injury. J Neurosurg Spine. 2012;17 (1 Suppl):119-28.
2. DiBardino DM, Wunderink RG. Aspiration pneumonia: a review of modern trends. J Crit Care. 2015;30:40-8.

3. Shin JC, Yoo JH, Lee YS, Goo HR, Kim DH. Dysphagia in cervical spinal cord injury. Spinal Cord. 2011;49:1008-13.

4. Winslow C, Bode RK, Felton D, Chen D, Meyer PR Jr. Impact of respiratory complications on length of stay and hospital costs in acute cervical spine injury. Chest. 2002;121:1548-54.

5. Hayashi T, Fujiwara Y, Sakai H, Maeda T, Ueta T, Shiba K. Risk factors for severe dysphagia in acute cervical spinal cord injury. Spinal Cord. 2017;55:940-3.

6. Ihalainen T, Luoto TM, Rinta-Kiikka I, Ronkainen A, Korpijaakko-Huuhka AM. Traumatic cervical spinal cord injury: recovery of penetration/aspiration and functional feeding outcome. Spinal Cord. 2018;56:1000-7.

7. Ihalainen T, Rinta-Kiikka I, Luoto TM, Koskinen EA, Korpijaakko-Huuhka AM, Ronkainen A. Traumatic cervical spinal cord injury: a prospective clinical study of laryngeal penetration and aspiration. Spinal Cord. 2017;55:979-84.

8. Ihalainen T, Rinta-Kiikka I, Luoto TM, Thesleff T, Helminen M, Korpijaakko-Huuhka AM, et al. Risk factors for laryngeal penetration-aspiration in patients with acute traumatic cervical spinal cord injury. Spine J. 2018;18:81-7.

9. Shem K, Castillo K, Wong S, Chang J. Dysphagia in individuals with tetraplegia: incidence and risk factors. J Spinal Cord Med. 2011;34:85-92.

10. Iruthayarajah J, McIntyre A, Mirkowski M, Welch-West P, Loh E, Teasell R. Risk factors for dysphagia after a spinal cord injury: a systematic review and meta-analysis. Spinal Cord. 2018; 56:1116-23.

11. Pongpipatpaiboon K, Inamoto Y, Aoyagi Y, Shibata S, Kagaya H, Matsuo K. Clinical Evaluation of Dysphagia. In: Saitoh E, Pongpipatpaiboon K, Inamoto Y, Kagaya H, editors. Dysphagia Evaluation and Treatment. 1st ed. Springer: Germany; 2018. p. 36-63.

12. Crary MA, Mann GD, Groher ME. Initial psychometric assessment of a functional oral intake scale for dysphagia in stroke patients. Arch Phys Med Rehabil. 2005;86:1516-20.

13. Kirshblum SC, Burns SP, Biering-Sorensen F, Donovan W, Graves DE, Jha A, et al. International standards for neurological classification of spinal cord injury (revised 2011). J Spinal Cord Med. 2011;34:535-46.

14. Abel R, Ruf S, Spahn B. Cervical spinal cord injury and deglutition disorders. Dysphagia. 2004;19:87-94.

15. Kirshblum S, Johnston MV, Brown J, O'Connor KC, Jarosz P. Predictors of dysphagia after spinal cord injury. Arch Phys Med Rehabil. 1999;80:1101-5.

16. Seidl RO, Nusser-Müller-Busch R, Kurzweil M, Niedeggen A. Dysphagia in acute tetraplegics: a retrospective study. Spinal Cord. 2010;48:197-201.

17. Wolf $\mathrm{C}$, Meiners TH. Dysphagia in patients with acute cervical spinal cord injury. Spinal Cord. 2003;41:347-53. 